\title{
A PARADOX OF LEWIS'S STRICT IMPLICATION
}

\author{
BY TANG TSAO-CHEN
}

The postulates for Lewis's strict implication are nine in number, ${ }^{*}$ namely,

[11.1]

$$
\begin{gathered}
p q-3 q p \\
p q-3 p \\
p-3 p p \\
(p q) r-\beta(q r) \\
p-3 \sim(\sim p) \\
p-3 q \cdot q-3 r:-3 \cdot p-3 r \\
p \cdot p-3 q:-\cdot q \\
\diamond p q-\diamond p \\
(\exists p, q): \sim(p-3 q) \cdot \sim(p-3 \sim q) .
\end{gathered}
$$

By the operations of substitution, adjunction, and inference, a body of theorems is obtained. But the following theorem, which is a paradox of the strict implication, is not explicitly mentioned in Lewis's book.

Any two of the first eight postulates are such that each is deducible from the other, if $p-3 q$ be interpreted as ' $p$ is deducible from $q . '$

In order to prove this theorem we assume the following eight theorems. $\dagger$

1.

$$
p \sim p=q \sim q
$$

Def.

$$
0=q \sim q
$$

* The references are to Symbolic Logic, by Lewis and Langford, 1932.

$\dagger$ For the proof of these theorems see the paper, The theorem " $p-3 q \cdot=\cdot p q$ $=p$ " and Huntington's relation between Lewis's strict implication and Boolean algebra, by Tang Tsao-Chen in this Bulletin, vol. 42 (1936), pp. 743-746. 
2.

3.

Def.

4.

5.

6.

7.

8.

$$
p \sim p=0
$$

$$
p 0=0
$$

$$
i=\sim \diamond 0
$$

$$
\begin{aligned}
& p q-3 p \cdot=. i \\
& p-3 p \cdot=. i \\
& p-3 q \cdot-3 \cdot i \\
& p-3 q \cdot=: i \cdot p-3 q \\
& p-3 q .=\cdot p q=p .
\end{aligned}
$$

Note that the Theorems 4 and 5 are particular cases of the following theorem.

9. If $p-3 q$ is asserted, then $p-3 q \cdot=\cdot i$.

$$
\begin{array}{lc}
\text { [Hyp.] } & p-3 q \\
{[(1), 8 .]} & p q=p \\
{[12.11]} & p q=p \cdot=\cdot p q=p \\
{[(2),(3)]} & p q=p \cdot=\cdot p=p \\
{[11.03,12.7]} & p=p \cdot=\cdot p-3 p \\
{[(4),(5), 5 .]} & p q=p \cdot=. i \\
{[(6), 8 .]} & p-3 q .=. i
\end{array}
$$

From the above theorem it is very easy to prove the following theorem.

10. If $p-3 q$ and $r-3 s$ are both asserted, then

$$
p-3 q .-3 . r-3 s
$$

and

$$
r-3 s \cdot 3 \cdot p-3 q \text {. }
$$

[Hyp.]

$$
p-3 q
$$

$[(3), 9$.

$$
p-3 q .=. i
$$

[Hyp.]

$$
r-3 s
$$




$$
\begin{array}{lc}
{[(5), 9 .]} & r-3 s .=. i \\
{[(4),(6)]} & p-3 q .=. r-3 s \\
{[11.03]} & (7)=(1)(2) \\
{[(7),(8)]} & (1)(2) \\
{[11.2]} & (1)(2)-3(1) \\
{[12.17]} & (1)(2)-3(2) \\
{[(9),(10)]} & (1) \\
{[(9),(11)]} & (2) .
\end{array}
$$

The paradox stated above is a particular case of Theorem 10, and therefore requires no further proof.

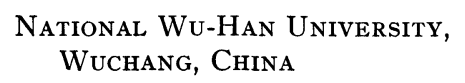

\title{
THE BETTI NUMBERS OF CYCLIC PRODUCTS
}

\author{
BY R. J. WALKER
}

1. Introduction. In a recent paper $\dagger \mathrm{M}$. Richardson has discussed the symmetric product of a simplicial complex and has obtained explicit formulas for the Betti numbers of the twoand three-fold products. Acting on a suggestion of Lefschetz, we define a more general type of topological product and apply Richardson's methods to compute the Betti numbers of a certain one of these, the "cyclic" product.

2. Basis for m-Cycles of General Products. Let $S$ be a topological space and $G$ a group of permutations on the numbers $1, \cdots, n$. The product of $S$ with respect to $G, G(S)$, is the set of all $n$-tuples $\left(P_{1}, \cdots, P_{n}\right)$ of points of $S$, where $\left(P_{i_{1}}, \cdots, P_{i_{n}}\right)$ is to be regarded as identical with $\left(P_{1}, \cdots, P_{n}\right)$ if and only if the permutation $\left(1_{i_{1} \ldots i_{n}}^{1} \ldots n_{n}\right)$ is an element of $G$. A neighborhood of $\left(P_{1}, \cdots, P_{n}\right)$ is the set of all points $\left(Q_{1}, \cdots, Q_{n}\right)$ for which $Q_{i}$ belongs to a fixed neighborhood of $P_{i}$. It is not difficult to verify that the

$\dagger$ M. Richardson, On the homology characters of symmetric products, Duke Mathematical Journal, vol. 1 (1935), pp. 50-69. We shall refer to this paper as $\mathrm{R}$. 